\title{
Evaluación de la cinética de crecimiento de las capas boruradas formadas sobre la superficie de un acero AISI 4150 endurecido a través del proceso borurización base polvo
}

\author{
Evaluation of the Growth Kinetics of Boride Layers Formed at the Surface in AISI 4150 \\ Borided Steel Using the Powder Pack Boriding Process
}

Martín Ortiz-Domínguez ${ }^{a}$, Arturo Cruz-Avilés ${ }^{b}$, Irving Morgado-González ${ }^{c}$, Jorge Zuno-Silva $^{d}$, Miguel Á. Flores-Rentería ${ }^{e}$, Milton C. Elías-Espinosa ${ }^{f}$, Rodolfo Velázquez-Mancilla $^{g}$

\begin{abstract}
:
In this study, the growth kinetics of boron on AISI 4150 steel by powder-pack boriding process was carried out. This superficial hardening technique produce the formation of a single-phase $\left(\mathrm{Fe}_{2} \mathrm{~B}\right)$. Thermochemical treatment for gray cast iron was made in the temperature range of $1123-1273 \mathrm{~K}$ for treatment times ranging from 2 to $8 \mathrm{~h}$. The boron diffusion coefficient in the $\mathrm{Fe}_{2} \mathrm{~B}$ layers was estimated by solving the mass balance equation $\left(\Delta C(d x / d t)_{x=\mathrm{v}=2 \varepsilon D_{\mathrm{Fe}_{2} t^{t / 2}}^{1 / 2}}=J_{\text {in }}^{\mathrm{Fe}_{2} B}(x)_{x=\mathrm{v}}-J_{\text {out }}^{F e}(x)_{x=\mathrm{v}+d \mathrm{v}}\right)$ at the $\left(\mathrm{Fe} \mathrm{e}_{2} \mathrm{~B} / \mathrm{substrate}\right)$ interface with an inclusion of boride incubation time $\left(t_{0}^{\mathrm{Fe}_{2} \mathrm{~B}}\right)$. The morphology of $\mathrm{Fe}_{2} \mathrm{~B}$ layers was observed by Optical Microscopy. Metallographic studies showed that the boride layer has a saw-tooth morphology in all the samples. The layer thickness measurements were done with the help of MSQ PLUS software. The $\mathrm{Fe}_{2} \mathrm{~B}$ phase was identified by X-ray diffraction method. Finally, the mathematical model was validated.
\end{abstract}

\section{Keywords:}

Activation energy, Boriding, Diffusion model, Growth kinetic

\section{Resumen:}

En el presente estudio se evalúa la cinética de difusión del boro en un acero AISI 4150 durante el proceso de borurización base polvo. Esta técnica de endurecimiento superficial produce en el material la formación de una fase característica, Fe2B. El tratamiento termoquímico se realizó en el rango de temperaturas de 1123-1273 K con tiempos de 2, 4, 6 y 8 h. Empleando la ecuación de balance

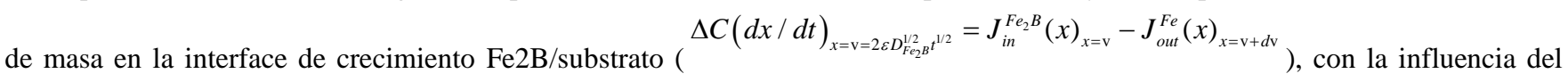
tiempo de incubación $\left({ }^{\mathrm{Fe}_{2} \mathrm{~B}}\right.$ ). La morfología de la capa Fe2B fue observada a través de Microscopía Óptica. Los estudios metalográficos mostraron que la capa borurada presenta una morfología aserrada en la interface de crecimiento para todas las muestras

\footnotetext{
a Autor de Correspondencia, Universidad Autónoma del Estado de Hidalgo, Escuela Superior de Ciudad Sahagún, ORCID: 0000-00034475-9804, Email: martin_ortiz@uaeh.edu.mx

b Universidad Autónoma del Estado de Hidalgo, Escuela Superior de Ciudad Sahagún, ORCID: 0000-0003-0455-1646, Email: arturo_cruz8085@uaeh.edu.mx

c Universidad Autónoma del Estado de Hidalgo, Escuela Superior de Ciudad Sahagún, Email: imorgadog@ hotmail.com

d Universidad Autónoma del Estado de Hidalgo, Escuela Superior de Ciudad Sahagún, Email: zunojorge@ gmail.com

e Universidad Autónoma del Estado de Hidalgo, Escuela Superior de Ciudad Sahagún, ORCID: 0000-0001-8018-4708, Email: miguel_flores10233@uaeh.edu.mx

${ }^{\mathrm{f}}$ Instituto Tecnológico de Estudios Superiores de Monterrey, Campus Santa Fe, Email: mielias@itesm.mx;

g Instituto Tecnológico de Tlalnepantla-ITTLA, Tlalnepantla de Baz, Email: itladepi20@ hotmail.com
} 
endurecidas. Los espesores de las capas fueron medidos con ayuda del software MSQ PLUS. La fase Fe2B fue identificada a través de la técnica difracción de rayos X (XRD). Finalmente, el modelo matemático fue validado.

\section{Palabras Clave:}

Energía de activación, Borurización, Modelo de difusión, Cinética de crecimiento

\section{Introducción}

Las actuales exigencias tecnológicas ponen de manifiesto la necesidad de disponer de materiales metálicos con características específicas, para condiciones de servicio cada vez más críticas. Así por ejemplo, las matrices metálicas empleadas en los procesos metalúrgicos del trabajado en frío y en caliente de los metales, necesitan de una alta tenacidad y elevada dureza superficial, especialmente a alta temperatura. El endurecimiento superficial de materiales metálicos como aceros, aleaciones, titanio, cobalto, níquel, etc; se puede conseguir, fundamentalmente, mediante dos procedimientos: modificando la composición química de la superficie mediante la difusión de algún elemento químico (carbono, nitrógeno, azufre, boro, aluminio, zinc, cromo, etc.) en cuyo caso se le conoce como tratamiento termoquímico, o modificando sólo la microestructura de la superficie por tratamiento térmico, conociéndose entonces como tratamiento térmico superficial. El impacto de los tratamientos superficiales y capas duras en nuestro país se refleja en el continuo establecimiento de nuevas industrias, centros tecnológicos y grupos de investigación que tratan de cubrir las necesidades del mercado. El estudio de todos estos procesos ha despertado un enorme interés en los últimos años, tanto a nivel académico como industrial, debido sobre todo al continuo crecimiento de las aplicaciones industriales. Entre los sectores industriales más beneficiados están, el sector mecánico y del automóvil, aeronáutico, energético, químico y bioquímico, electrónico, biomédico, manufacturero, etc.

Los tratamientos termoquímicos aplicados al acero son aquellos en los cuales la composición de la superficie de la pieza se altera por la adición de carbono, nitrógeno, boro u otros elementos. Los tratamientos más comunes en la industria internacional son: carburización, nitruración, y borurización. Estos tratamientos implican una alteración de la composición química de la capa superficial del metal tratado, debido a la difusión de átomos de un medio circundante hacia el interior del metal, y dependen de varios factores, como son: temperatura, tiempo, concentración del medio circundante, etc., Así como del control de las variables anteriores. Los tratamientos termoquímicos se utilizan cuando se requiere asegurar una determinada profundidad y zonificación de la capa endurecida. El carbono, el nitrógeno y el boro, en razón de su pequeño tamaño (capaces de ser alojados en huecos intersticiales), son los elementos más usados para endurecer un acero, por lo que se utilizan ampliamente en este tipo de tratamientos.

El tratamiento termoquímico de borurización en polvo es un tratamiento de tipo superficial el cual consta de la difusión de átomos de boro en un substrato con el fin de generar una capa denominada $\mathrm{Fe}_{2} \mathrm{~B}$, la cual presenta mejoras en sus propiedades como: dureza, resistencia al desgaste, altas temperaturas de fusión, entre otras. El principal sector de aplicación para el tratamiento de borurización es el sector industrial, debido a las mejoras en las propiedades de los componentes de máquinas y por ende obteniéndose un mayor tiempo de vida útil. 1-3 La borurización se puede aplicar en diferentes aleaciones tanto ferrosas como no ferrosas, donde el factor limitante es la solubilidad del boro con los elementos presentes en el substrato. Algunos elementos químicos que no son solubles con el boro son: aluminio, carbono y silicio, por lo tanto, las aleaciones con alto contenido en peso de estos elementos químicos no son recomendable borurizarlas por la variación de las propiedades esperadas. Los modelos de difusión han pasado por una constante evolución, comenzando desde la llegada de la borurización a México en el año de 1997, y posteriormente con la mejora por la interpretación matemática del crecimiento de los boruros. Al inicio no se consideraban los periodos de incubación y posteriormente con el desarrollo de investigación se consideraron. En la siguiente sección se muestra el desarrollo matemático del crecimiento de la capa $\mathrm{Fe}_{2} \mathrm{~B}$ en el acero AISI 4150 basado en la resolución de las Leyes de Fick, utilizando un medio de saturación en polvo. 3-5

\section{Modelo de difusión}

El modelo de difusión es una descripción las etapas involucradas a lo largo del proceso de borurización en polvo, iniciando, con el crecimiento de la capa $\mathrm{Fe}_{2} \mathrm{~B}$ en la superficie del substrato, posteriormente, realizando un balance de masa en la interface ( $\mathrm{Fe}_{2} \mathrm{~B} /$ substrato) debido a la diferencia notable de concentraciones. El modelo de difusión estacionario describe como crece la capa $\mathrm{Fe}_{2} \mathrm{~B}$ en la superficie del substrato sin la consideración del tiempo 
$(t=0)$. Al iniciar el proceso de borurización en el tiempo cero, como se muestra en la Figura (1), tanto el substrato como el polvo de borurización (carburo de silicio, tetraflouroborato de potasio y carburo de boro) están en contacto directo y aun no existe un crecimiento de capa.

Para iniciar el crecimiento de capa debe existir un tiempo de incubación $\left(t_{0}^{\mathrm{Fe}_{2} B}\right)$, el cual inicia en $(t=0)$ y termina en $t_{0}^{F e_{2} B}$ (ver Figura (1)). Una vez que la incubación termina, inicia el proceso de crecimiento de la capa $\mathrm{Fe}_{2} \mathrm{~B}$ desde la superficie del substrato y manejando una concentración de boro $C_{u p}^{F_{e} B}$ hacia el interior del substrato, finalizando con una concentración de boro $C_{l o w}^{F_{e} B}$.

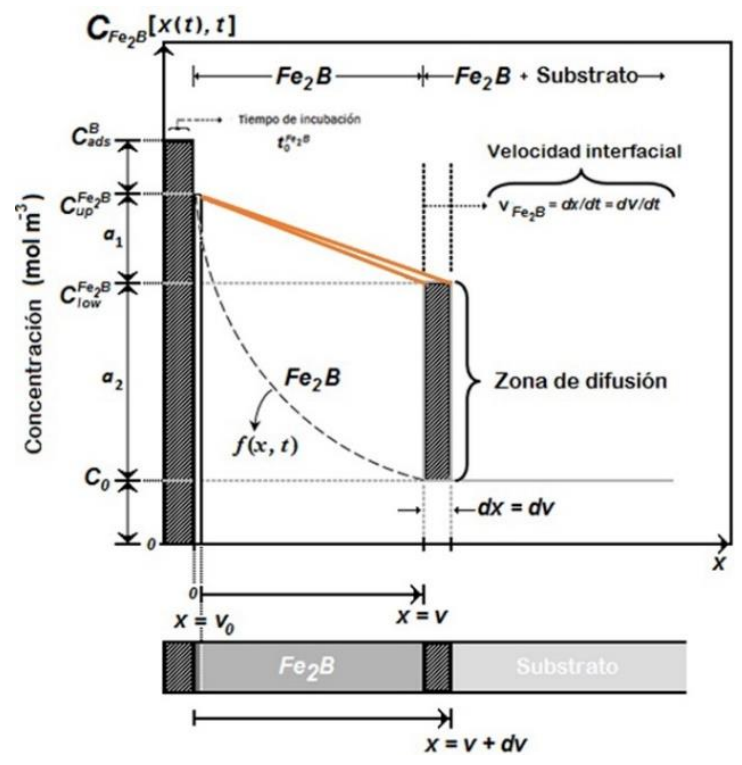

Figura 1. Perfil de concentración de la capa $\mathrm{Fe}_{2} \mathrm{~B}$.

(Elaboración Propia)

De tal modo que la capa $\mathrm{Fe}_{2} \mathrm{~B}$ obtiene un espesor (v) donde la concentración de boro es mayor en la superficie que en el interior del substrato. El término $C_{a d s}^{B}$ representa la concentración efectiva de boro.

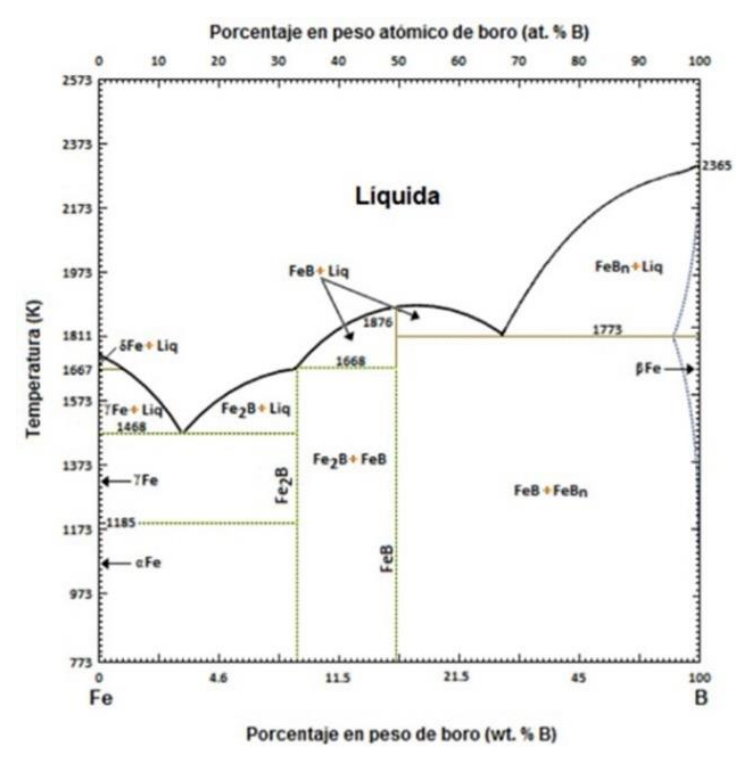

(Elaboración Propia)

Figura 2. Diagrama de Fase Hierro-Boro.

El diagrama de fase Fe-B que se muestra en la Figura (2), muestra la transformación de hierro respecto a los átomos de boro. En la zona izquierda muestra como los átomos de boro se van difundiendo en el substrato (hierro) para formar $\alpha-\mathrm{Fe}-\mathrm{Fe}_{2} \mathrm{~B}$. La fase $\mathrm{Fe}_{2} \mathrm{~B}$ formada en la superficie presenta un porcentaje de 8.83 de peso en boro. 6

Para determinar los coeficientes de difusión $\left(D_{\mathrm{Fe}_{2} B}\right)$ es necesario conocer las condiciones a la frontera que se manejan en el perfil de crecimiento de la capa $\mathrm{Fe}_{2} \mathrm{~B}$ las cuales son:

$$
\begin{gathered}
C_{\mathrm{Fe}_{2} B}\left(x=\mathrm{v}_{0}\right)=C_{u p}^{\mathrm{Fe}_{2} B},(1) \\
C_{\mathrm{Fe}_{2} B}(x=\mathrm{v})=C_{l o w}^{\mathrm{Fe}_{2} B} .
\end{gathered}
$$

Para determinar el perfil de concentración del boro a lo largo de la fase $\mathrm{Fe}_{2} \mathrm{~B}$, se parte de la segunda ley de Fick:

$$
\frac{\partial C_{\mathrm{Fe}_{2} B}(x, t)}{\partial t}=D_{\mathrm{Fe}_{2} B} \frac{\partial^{2} C_{\mathrm{Fe}_{2} B}(x, t)}{\partial x^{2}}
$$

Considerando el estado estacionario donde $(t=0)$, por lo tanto, la Ecuación (3) se transforma a una ecuación diferencial ordinaria de segundo orden.

$$
\frac{d^{2} C_{F_{2} B}(x)}{d x^{2}}=0 .
$$


Se realiza una separación de variables y posteriormente se integra la Ecuación (4) para así encontrar la primera constante de integración $\left(c_{1}\right)$.

$$
\frac{d C_{\mathrm{Fe}_{2} B}(x)}{d x}=c_{1} .
$$

Posteriormente, se aplica una segunda separación de variables y se integra la Ecuación (5), al realizar esta operación se determina un perfil de concentración de boro para el caso estacionario de la fase $\mathrm{Fe}_{2} \mathrm{~B}$ en función de dos constantes de integración $\left(c_{1}\right)$ y $\left(c_{2}\right)$.

$$
C_{F e_{2} B}(x)=c_{1} x+c_{2} .
$$

Sustituyendo la condición de frontera de la Ecuación (1) en la ecuación del perfil de concentración de la capa $\mathrm{Fe}_{2} \mathrm{~B}$ en la Ecuación (6), se obtiene $\left(c_{2}\right)$.

$$
\left(C_{2}\right)=C_{u p}^{F e_{2} B} .
$$

La sustitución de la Ecuación (7) en el perfil de concentración de la capa $\mathrm{Fe}_{2} \mathrm{~B}$ queda se la siguiente manera:

$$
C_{\mathrm{Fe}_{2} B}(x)=c_{1} x+C_{u p}^{\mathrm{Fe}_{2} B} .
$$

Se sustituye la segunda condición de frontera de la Ecuación (2) en la Ecuación (8) y se despeja la primera constante de integración $\left(c_{1}\right)$.

$$
C_{1}=\frac{C_{l o w}^{F e_{2} B}+C_{u p}^{F e_{2} B}}{\mathrm{v}} .
$$

Por último, el valor obtenido de la constante de integración $\left(c_{1}\right)$, (Ecuación (9)) y el valor obtenido de la constante de integración $\left(c_{2}\right)$ (Ecuación (7)), se sustituyen en la Ecuación (6) obteniendo lo siguiente:

$$
C_{F_{2} B}(x)=\frac{C_{l o w}^{F e_{2} B}+C_{u p}^{F e_{2} B}}{\mathrm{v}} x+C_{u p}^{F e_{2} B} .
$$

Una vez encontrado el perfil de crecimiento de la capa $\mathrm{Fe}_{2} \mathrm{~B}$, se realiza una evaluación de flujos ( $\left(\begin{array}{lll}J_{\text {in }} \mathrm{Fe}_{2} B & \text { y } & J_{\text {out }} \mathrm{Fe}_{2} \mathrm{~B}\end{array}\right)$ en dónde exista una diferencia notable de concentraciones. La evaluación de los flujos se denomina balance de masa el cual se aplica en una sección denominada $(d \mathrm{v})$. (Ver Figura (3)).

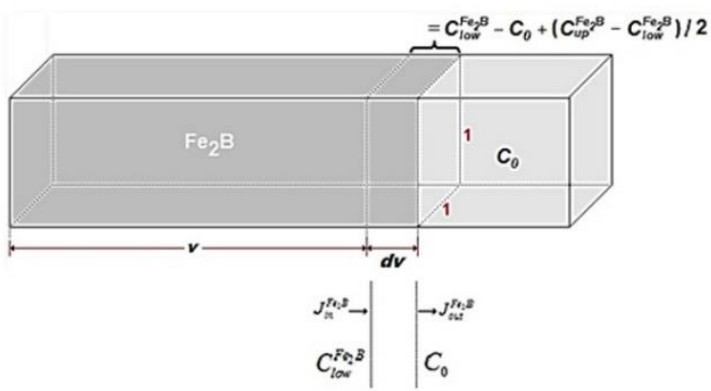

Figura 3. Balance de masa ( $\mathrm{Fe}_{2} \mathrm{~B} /$ substrato).

(Elaboración Propia)

En la Figura (3) se puede observar que el área denominada $(d \mathrm{v})$ perteneciente a una sección transversal de una probeta de acero AISI 4150, es la interface entre $\mathrm{Fe}_{2} \mathrm{~B}$ y el substrato. El balance de masa se basa en la relación de los átomos de boro que entran (flujo de entrada) menos los átomos de boro que salen (flujo de salida) donde ambos flujos se evalúan de la siguiente manera.

$$
\begin{aligned}
& \left.J_{\text {in }}^{F e_{2} B}\right|_{x=\mathrm{v}}, \\
& \left.J_{\text {out }}^{F e_{2} B}\right|_{x=\mathrm{v}+d v} .
\end{aligned}
$$

La concentración de átomos en la interface $(d \mathrm{v})$ se puede representar usando las Ecuaciones ((11) y (12)).

$$
\begin{aligned}
& C_{\text {low }}^{\mathrm{Fe}_{2} B}-C_{0}+\left(C_{\text {up }}^{\mathrm{Fe}_{2} B}-C_{\text {low }}^{\mathrm{Fe}_{2} B}\right) / 2(d \mathrm{v} / d t)= \\
& \left.J_{\text {in }}^{\mathrm{Fe}_{2} B}\right|_{x=\mathrm{v}}-\left.J_{\text {out }}^{\mathrm{Fe}_{2} B}\right|_{x=\mathrm{v}+d \mathrm{v}} .
\end{aligned}
$$

Se sabe que existe un flujo de átomos de boro hacia la interface que son representados por:

$$
\begin{aligned}
& \left.J_{i n}^{F e_{2} B}\right|_{x=v}=-D_{F_{2} B} \frac{d C_{F_{2} B}(x)}{d x}, \\
& \left.J_{\text {out }}^{F e_{2} B}\right|_{x=v+d v}=-D_{F_{2} B} \frac{d C_{F_{2} B}(x)}{d x} .
\end{aligned}
$$

Sustituyendo las Ecuaciones ((14) y (15)) en la Ecuación (13) se tiene:

$$
\begin{aligned}
& C_{l o w}^{F e_{2} B}-C_{0}+\left(C_{u p}^{\mathrm{Fe}_{2} B}-C_{l o w}^{\mathrm{Fe}_{2} B}\right) / 2(d \mathrm{v} / d t)= \\
& \left.J_{i n}^{F e_{2} B}\right|_{x=\mathrm{v}}=-D_{\mathrm{Fe}_{2} B} \frac{d C_{\mathrm{Fe}_{2} B}(x)}{d x} .
\end{aligned}
$$


Con, $\left.J_{\text {out }}^{\mathrm{Fe}_{2} B}\right|_{x=\mathrm{v}+d \mathrm{v}} \approx 0$. Existe una relación respecto al flujo y el gradiente de concentración que está dada por la primera ley de Fick.

$$
J_{\mathrm{Fe}_{2} B}(x)=D_{\mathrm{Fe}_{2} B}\left(\frac{C_{u p}^{\mathrm{Fe}_{2} \mathrm{~B}}-\mathrm{C}_{\mathrm{low}}^{\mathrm{Fe}_{2} \mathrm{~B}}}{\mathrm{v}}\right) .
$$

La Ecuación (17) es posible sustituirla en la Ecuación (16) para obtener lo siguiente:

$$
\begin{aligned}
& C_{l o w}^{\mathrm{Fe}_{2} B}-C_{0}+\left(\left(C_{u p}^{\mathrm{Fe}_{2} B}-C_{l o w}^{F e_{2} B}\right) / 2\right)(d \mathrm{v} / d t)= \\
& D_{\mathrm{Fe}_{2} B}\left(\frac{C_{u p}^{F e_{2} B}-C_{l o w}^{F e_{2} B}}{\mathrm{v}}\right) .
\end{aligned}
$$

La Ecuación (18) es una ecuación diferencial ordinaria de primer orden. Separando términos e integrando ambos lados, se tiene:

$$
\int_{\mathrm{v}=\mathrm{v}_{0}}^{\mathrm{v}=\mathrm{v}} \mathrm{v} d \mathrm{v}=2 D_{\mathrm{Fe}_{2} B}\left(\frac{C_{u p}^{F e_{2} B}-C_{l o w}^{F e_{2} B}}{C_{l o w}^{F e_{2} B}-2 C_{0}+C_{u p}^{F e_{2} B}}\right)_{t=t_{0}}^{t=t} d t
$$

Una vez integrados ambos términos de la Ecuación (19) y despejando el espesor de capa (v), se determina la ecuación de la ley de crecimiento parabólico, como se presenta en la Ecuación (20):

$$
\mathrm{v}^{2}=4 D_{\mathrm{Fe}_{2} B} \varepsilon^{2} t
$$

Con $\varepsilon^{2}=C_{u p}^{\mathrm{Fe}_{2} B}-C_{l o w}^{\mathrm{Fe}_{2} \mathrm{~B}} / C_{l o w}^{\mathrm{Fe}_{2} \mathrm{~B}}-2 C_{0}+C_{u p}^{\mathrm{Fe}_{2} B}$. De la Ecuación (20) es posible determinar los coeficientes de difusión de boro $\left(D_{\mathrm{Fe}_{2} \mathrm{~B}}\right.$ ) respectivos a cada temperatura, graficando el espesor al cuadrado respecto al tiempo ( $\mathrm{v}^{2}$ vs $\left.t\right)$ (ver Figura 4).

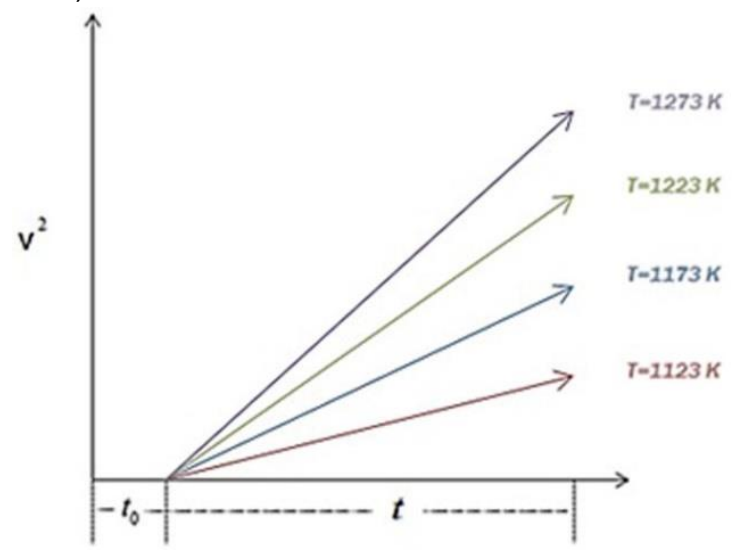

Figura 4. Pendientes relativas respecto a cada temperatura de tratamiento.

(Elaboración propia)
Además, es necesario calcular un coeficiente de difusión generalizado y específico para el material, esto es posible, analizando si existe un incremento de la pendiente respecto al aumento de temperatura, al ser así, se generaliza la Ecuación (20) de acuerdo a un comportamiento tipo Arrhenius quedando de la siguiente manera.

$$
\mathrm{v}=2 D_{0}^{1 / 2} \varepsilon_{\mathrm{Fe}_{2} B} e^{-Q / R T} t^{1 / 2}
$$

\section{Procedimiento experimental}

Se empleó un acero AISI 4150, el cual tiene una composición química que se muestra en la Tabla (1). Este acero es comúnmente utilizado en la fabricación de moldes para inyección de plástico y para moldes de fundición de zinc. Una de sus principales características es la excelente pulibilidad y buen texturizado, lo que le provee una amplia cantidad de aplicaciones; en implementos de maquinaria, moldes, piezas de apoyo y sujeción, etc. 7,8

\begin{tabular}{cccccccc}
\hline Elemento quimico & $\mathbf{C}$ & Si & P & Cr & Mo & Mn & S \\
\hline \% PESO & $0.48-53$ & $0.20-0.35$ & 0.035 & $0.80-1.1$ & $0.15-0.25$ & $0.75-1.00$ & 0.040 \\
\hline
\end{tabular}

Tabla 1. Composición química del acero AISI 4150.

Como primer paso, se cortó una pequeña sección de un lingote de acero AISI 4150 con una cortadora de la marca

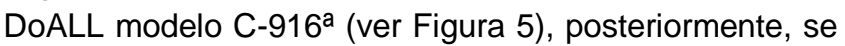
cortaron veinte probetas de forma cúbica con dimensiones de un centímetro por lado como se puede apreciar en la Figura 6.

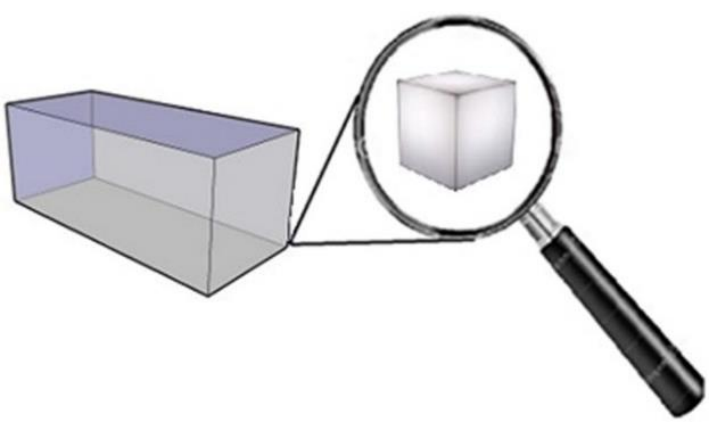

Figura 5. Sección del lingote de acero AISI 4150.

(Elaboración Propia)

Las probetas se montaron en dispositivos especiales para obtener una mejor sujeción y manipulación de éstas. Al finalizar el montaje, se inició el proceso de desbaste grueso aplicado a lo largo de las secciones donde se 
evaluará el tratamiento, utilizando una lijadora de banda de la marca Buehler (ver Figura 7). 7

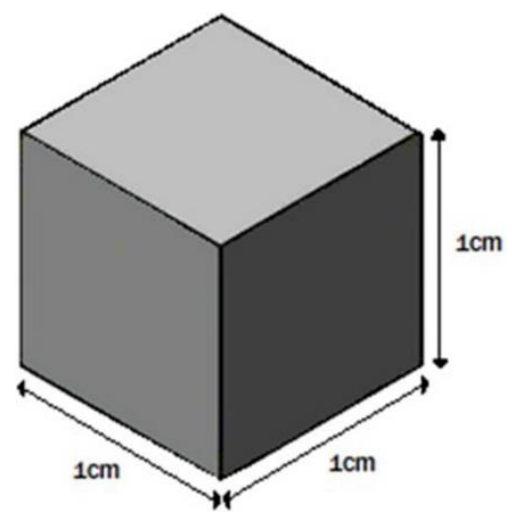

Figura 6. Probeta de acero AISI 4150.

(Elaboración Propia)

Al término del desbaste grueso, se inició el proceso de desbaste delgado utilizando lijas de carburo de silicio con diferentes granulometrías (80-2000), esto se hizo con el fin de garantizar una sección de la muestra limpia y con pocas imperfecciones debido al maquinado de las probetas. 9

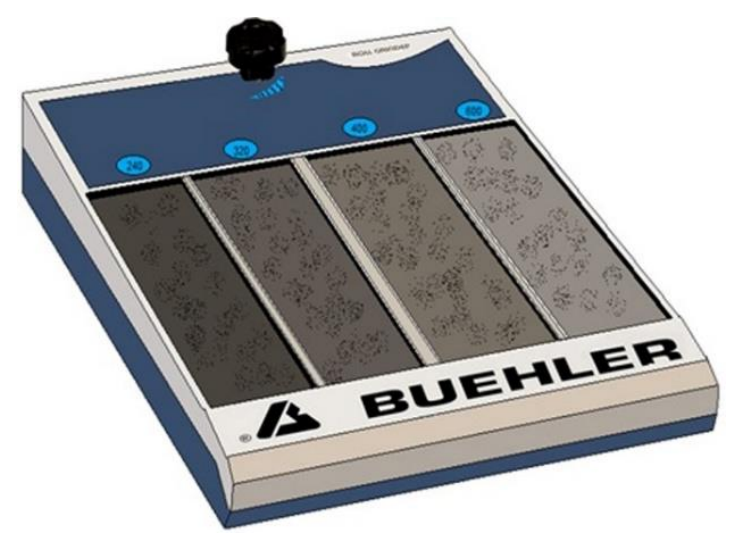

Figura 7. Pulidora Buehler.

(Elaboración Propia)

Una vez terminado el proceso de desbaste delgado, las probetas se lavan con agua para limpiar la superficie y se introducen en alcohol etílico para ser secadas rápidamente y evitar indicios de corrosión. Finalmente, las muestras se desmontan teniendo cuidado de evitar cualquier contacto, disminuyendo así la probabilidad de rayado. El tratamiento termoquímico de borurización en

*AISI 316L Acero utilizado para el manejo de productos químicos y equipos de manejo de alimentos.

†Polvo para borurizar: $\mathrm{KBF}_{4}+\mathrm{SiC}+\mathrm{B}_{4} \mathrm{C}$. Compuestos químicos contenidos en el medio borurante. polvo es una técnica que utiliza tetraflouroborato de potasio $\left(\mathrm{KBF}_{4}\right)$, carburo de silicio (SiC), carburo de boro $\left(\mathrm{B}_{4} \mathrm{C}\right)$ y oxígeno $\left(\mathrm{O}_{2}\right)$ el cual se encuentra presente en la atmósfera donde se aplique el tratamiento. Las probetas mencionadas anteriormente, se introducen en pequeños contenedores fabricados de acero AISI $316 L^{*}$ y con dimensiones superiores de acuerdo al tamaño de las probetas como se puede apreciar en la Figura 8.
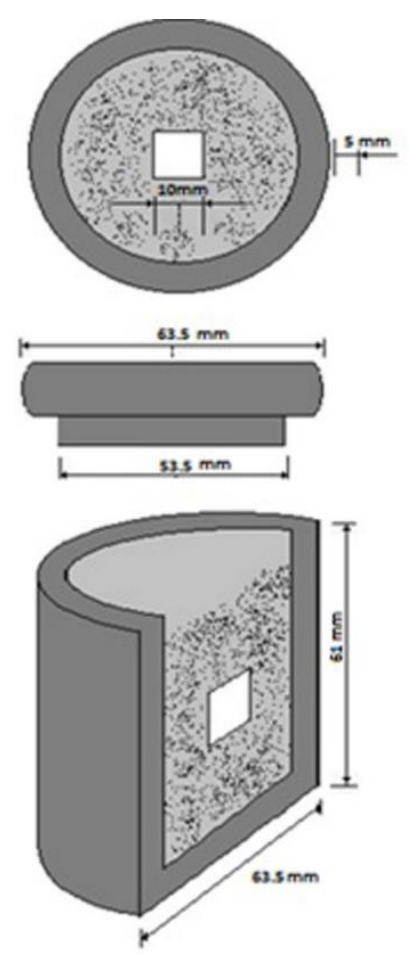

Figura 8. Contenedor para tratamiento de borurización.

(Elaboración Propia)

El siguiente paso para el tratamiento de borurización, es colocar una base de polvo para borurizar ${ }^{\dagger}$ aproximadamente de un centímetro de espesor dentro del contenedor, posteriormente se coloca la probeta sobre del polvo de borurizar, se embebe totalmente y se coloca su tapa de tal modo que ajuste correctamente. Dentro de los contenedores se pueden llevar a cabo dos reacciones de acuerdo a la cantidad de oxígeno presente en la atmósfera;

$$
\begin{aligned}
& \mathrm{B}_{4} \mathrm{C}+4 \mathrm{KBF}_{4}+3 \mathrm{SiC}+2 \mathrm{O}_{2} \rightarrow 8 \mathrm{~B}+4 \mathrm{KF}+3 \mathrm{SiF}_{4} \\
&+4 \mathrm{CO} .(22) \\
& \mathrm{B}_{4} \mathrm{C}+4 \mathrm{KBF}_{4}+3 \mathrm{SiC}+ 3 \mathrm{O}_{2} \rightarrow 6 \mathrm{~B}+4 \mathrm{KF}+3 \mathrm{SiF}_{4}+4 \mathrm{CO} \\
&+ \mathrm{B}_{2} \mathrm{O}_{2} .(23)
\end{aligned}
$$


Las ecuaciones químicas anteriores muestran como varia la concentración de boro dependiendo de la cantidad presente de oxígeno en la atmósfera, en la Ecuación (22) se observa, el contenido de cuatro átomos de oxígeno en forma gaseosa y se liberan ocho átomos de boro en forma gaseosa, mientras que en la Ecuación (23) se tienen seis átomos de oxígeno y se liberan seis átomos de boro. Esto significa, a mayor presencia de oxígeno en la atmósfera, la oxidación ocurre más fácilmente y disminuye la concentración de boro en forma gaseosa. Debido a lo anterior, es recomendable que la tapa del contenedor ajuste perfectamente para evitar el exceso de oxígeno en el interior del contenedor. El modo más adecuado para obtener la máxima efectividad del tratamiento es utilizar una atmósfera controlada de argón para evitar la oxidación. Continuando con el proceso de borurización, las probetas se introducen en los contenedores y se preparan para ser introducidas en una mufla de la marca Carbolite modelo RFW1200 con atmósfera controlada de argón, considerando un tiempo de incubación $\left({ }^{\mathrm{Fe}_{2} \mathrm{~B}}\right)$ aproximado de treinta minutos. El horno se mantiene en un rango de temperatura de $(1123$ - $1273 \mathrm{~K})$ con una corriente continua de gas (argón) y únicamente se toman en cuenta los tiempos de borurización (2, 4, 6 y 8 h) (ver Figura 9). 10

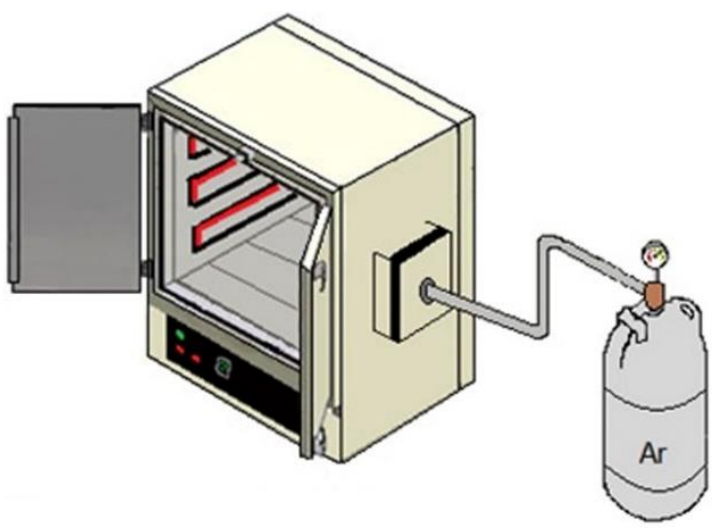

Figura 9. Mufla con atmósfera controlada para tratamiento termoquímico.

(Elaboración Propia)

Posteriormente, al término del tiempo de tratamiento, los contenedores son retirados de la mufla y se dejan enfriar al aire libre sin utilizar algún otro método de enfriado, esto se hace con el fin de evitar desprendimientos de capa debido a choques térmicos. Finalmente, las muestras se extraen de los contenedores y se inicia la preparación metalográfica de las probetas. 11

\section{Preparación metalográfica}

Las probetas de acero AISI 4150 se montaron en dispositivos especiales (ver Figura (10)), los cuales poseen varios objetivos; mejor manipulación, mayor agarre y evitar la formación de caras en la probeta al momento del desbaste, la dureza que presentan las placas de montaje es mayor en comparación a la baquelita, ya que éste es otro material comúnmente usado para el montaje de muestras metalográficas, por lo tanto, se evita la formación de caras. Otro aspecto importante es la colocación de la probeta dentro del dispositivo de montaje, primeramente, se utilizan unas placas de cobre y aluminio que se insertan entre la probeta y las placas de montaje con el fin de evitar deformaciones en la probeta por la presión ejercida al cerrar los prisioneros del dispositivo.

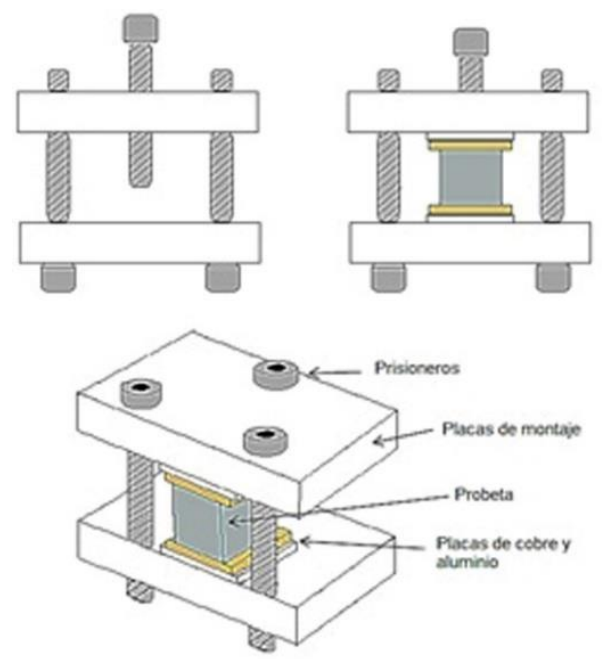

Figura 10. Dispositivo montador de probetas.

(Elaboración Propia)

Al término del montaje de las probetas, se lleva a cabo una selección de lijas de carburo de silicio llevando una secuencia de acuerdo al tamaño de grano, iniciando con el número $80,120,180,220,240,280,320,360,400,600$, $800,1000,1500$ y 2000. Posteriormente, se inicia el proceso de desbaste en el que se utilizó una pulidora automática de la marca Buehler modelo Phoenix Beta (ver Figura 11). 


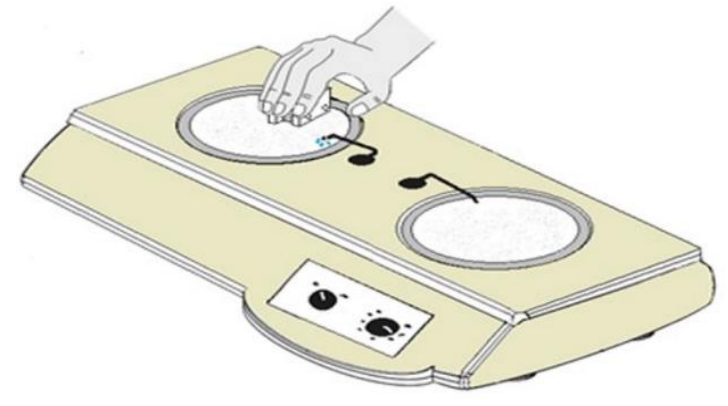

Figura 11. Pulidora Phoenix Beta.

(Elaboración Propia)

Al finalizar el proceso de desbaste, se inicia el proceso de pulido, utilizando paño para pulir y alúmina de diferentes tamaños de partícula $(5.0,1,0,0.3 \mu \mathrm{m})$ durante un tiempo aproximado de sesenta minutos con el fin de eliminar cualquier ralladura y obtener un acabado espejo. Posteriormente, las probetas se desmontan de los dispositivos de montaje y continúa la revelación de la fase $\mathrm{Fe}_{2} \mathrm{~B}$ a partir de un ataque químico como se aprecia en la Figura 12.

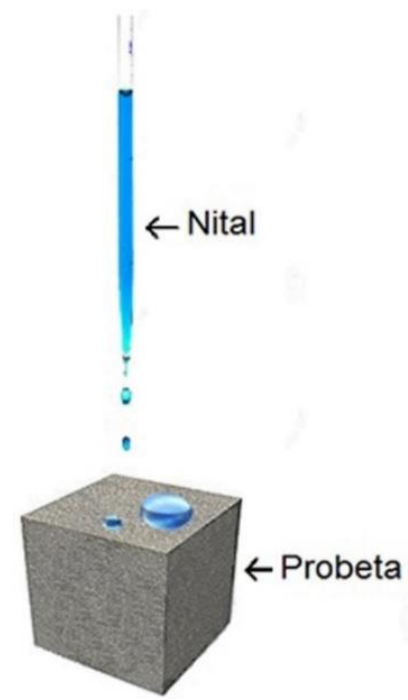

Figura 12. Ataque químico.

(Elaboración Propia)

El objetivo del ataque químico es revelar la microestructura incluyendo defectos y fases. El ataque químico utilizado fue nital, el cual contiene ácido nítrico $2 \%$ y etanol $98 \%$, este tipo de ataque es comúnmente utilizado en la revelación de hierros, aceros al carbón y aceros aleados. Una vez desmontadas las probetas, se les coloca unas gotas de nital en la superficie que se desea revelar durante cinco segundos, posteriormente se lava rápidamente con abundante agua para evitar un sobre ataque, seguido a esto, la misma probeta se enjuaga con alcohol etílico y se seca con una corriente de aire caliente para evitar indicios de corrosión. Finalmente, el crecimiento de la capa $\mathrm{Fe}_{2} \mathrm{~B}$, se observa en un microscopio metalográfico de la marca Olympus modelo GX71, en el que cuenta con cámara fotográfica para la captura de imágenes (ver Figura 13). 11

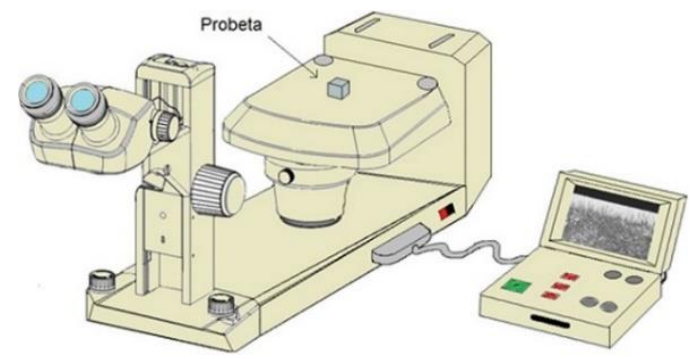

Figura 13. Microscopio metalográfico marca Olympus GX51.

(Elaboración Propia)

\section{Difracción de rayos $X$}

Con el propósito de verificar la fase presente $\left(\mathrm{Fe}_{2} \mathrm{~B}\right)$ sobre la capa borurada. Se realizó un análisis por Difracción de rayos $\mathrm{X}$ aplicado sobre la probeta borurada de acero AISI 4150 .

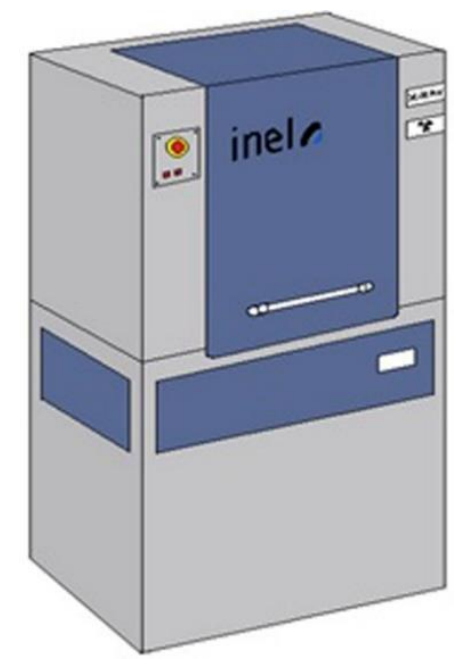

Figura 14. Difractómetro Inel, modelo EQUINOX 2000.

(Elaboración Propia)

Se empleó una radiación con una lámpara de cobalto ( $\mathrm{CoK}_{\mathrm{a}}$ ) con una longitud de onda $1.78 \AA$, utilizando un difractómetro de la marca Inel modelo EQUINOX 2000 (ver Figura 14). En la Figura (15) se muestra como los rayos $X$ inciden sobre una muestra. Los rayos $X$ viajan desde el tubo de rayos $X$ hasta la muestra, estos rayos chocan con los átomos superiores de la probeta y reemiten una radiación formando un ángulo. Existen ángulos privilegiados formados entre el haz incidente y el 
difractado en el que la emisión de radiación se encuentra en fase y satisface la Ley de Bragg. 12

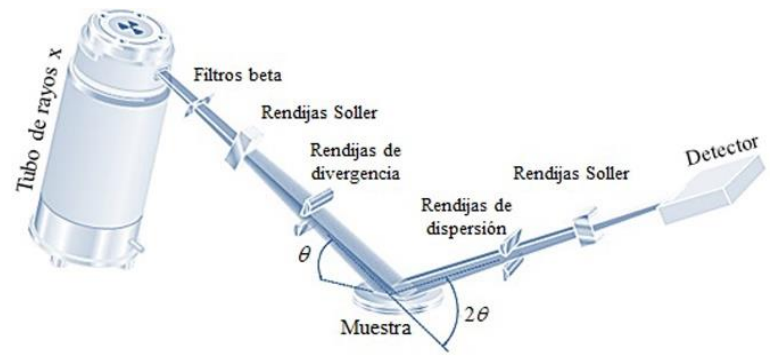

Figura 15. Esquematización del funcionamiento de un difractómetro de rayos $X$.

(Elaboración Propia)

\section{Resultados y discusión}

En las Figuras 16, 17, 18 y 19, se presentan las micrografías del acero borurado AISI 4150 con la técnica de borurización de empaquetamiento en caja, para diferentes temperaturas y tiempos de tratamiento.

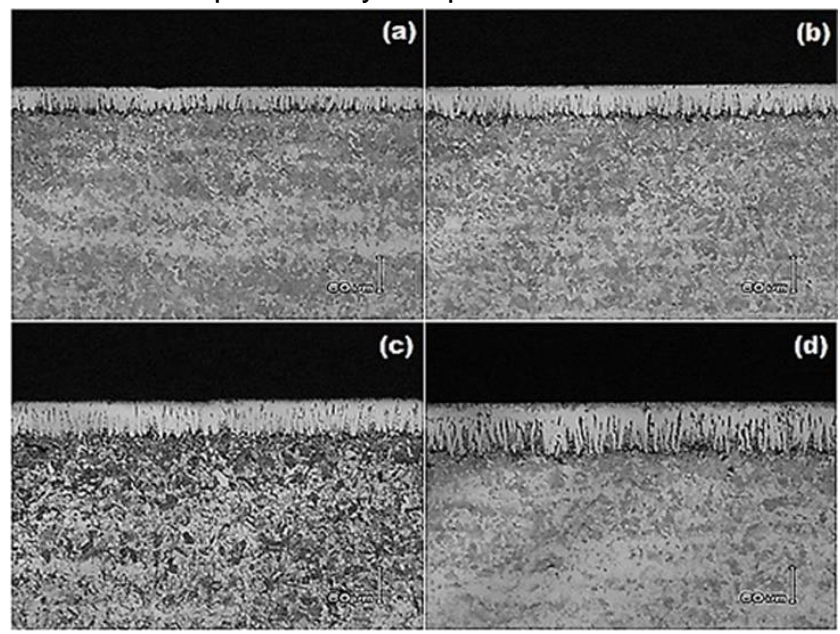

Figura 16. Morfología aserrada de la capa $\mathrm{Fe}_{2} \mathrm{~B}$ formada en la superficie del acero AISI 4150 a una temperatura de 1123

$K, a) 2$ de tiempo de tratamiento; $b$ ) con $4 \mathrm{~h}$ de tratamiento;

c) $6 \mathrm{~h}$ de tiempo de tratamiento $y \mathrm{~d}$ ) $8 \mathrm{~h}$ de tiempo de tratamiento.

(Elaboración Propia)

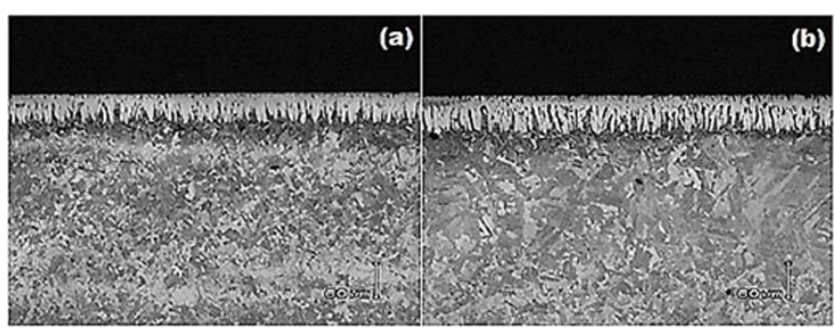

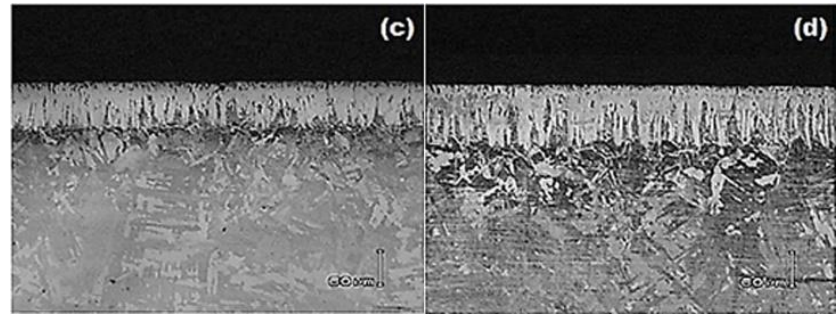

Figura 17. Morfología aserrada de la capa $\mathrm{Fe}_{2} \mathrm{~B}$ formada en la superficie del acero AISI 4150 a una temperatura de 1173 $K, a) 2 \mathrm{~h}$ de tiempo de tratamiento; $b$ ) con $4 \mathrm{~h}$ de tratamiento; c) 6 h de tiempo de tratamiento y d) 8 h de tiempo de tratamiento.

(Elaboración Propia)

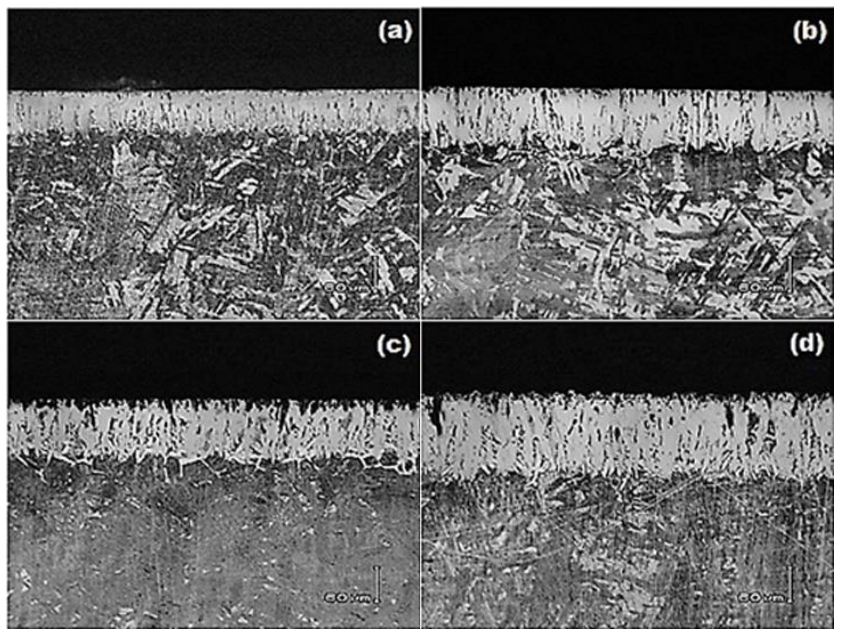

Figura 18. Morfología aserrada de la capa $\mathrm{Fe}_{2} \mathrm{~B}$ formada en la superficie del acero AISI 4150 a una temperatura de 1223 $K, a) 2$ de tiempo de tratamiento; b) con 4 h de tratamiento; c) $6 \mathrm{~h}$ de tiempo de tratamiento $y \mathrm{~d}$ ) con $8 \mathrm{~h}$ de tratamiento.

(Elaboración Propia)

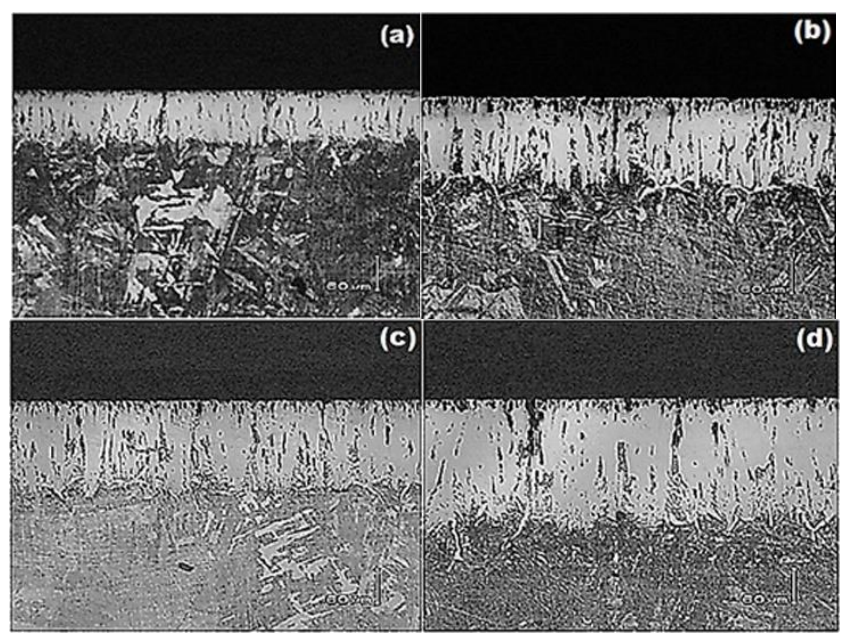


Figura 19. Morfología aserrada de la capa $\mathrm{Fe}_{2} \mathrm{~B}$ formada en la superficie del acero AISI 4150 a una temperatura de 1273

$K$, a) 2 h de tiempo de tratamiento; $b)$ con $4 h$ de tratamiento; c) $6 \mathrm{~h}$ de tiempo de tratamiento y d) con $8 \mathrm{~h}$ de tratamiento.

(Elaboración Propia)

Para verificar la presencia de las fases $\mathrm{Fe}_{2} \mathrm{~B}$ sobre la superficie de los aceros, se realizó un análisis por difracción de rayos $X$, aplicando sobre la superficie de la pieza borurada radiación CoKa con una longitud de onda de $1.7889 \AA$ A. En la Figura 20, se comprueba la presencia de la fase $\mathrm{Fe}_{2} \mathrm{~B}$.

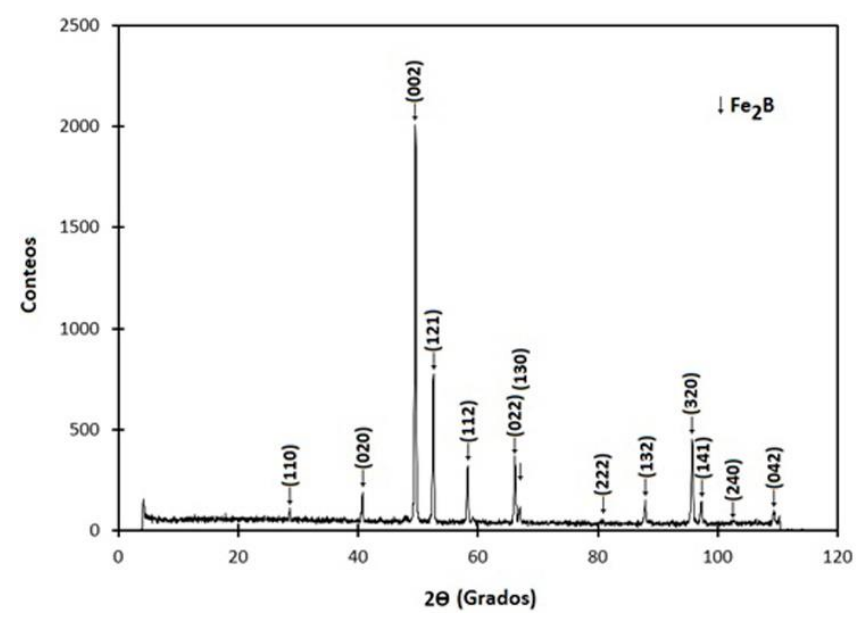

Figura 20. Patrón de difracción de rayos $X$ originado durante la detección de la fase $\mathrm{Fe}_{2} \mathrm{~B}$ en la superficie de la AISI 4150 con la temperatura de tratamiento de $1273 \mathrm{~K}$ y $8 \mathrm{~h}$ de tiempo de tratamiento.

(Elaboración Propia)

Continuando con el análisis de los resultados, en la Tabla (2) se muestran los espesores obtenidos de la medición de la capa borurada $\mathrm{Fe}_{2} \mathrm{~B}$ para el proceso de borurización en polvo de acuerdo a los diferentes parámetros del tratamiento de borurización.

\begin{tabular}{|c|c|c|c|c|}
\hline \multirow{2}{*}{ Temperatura } & \multicolumn{4}{|c|}{$\begin{array}{c}\text { Espesor de capa } \\
(\mu \mathrm{m})\end{array}$} \\
\cline { 2 - 5 } & $2 \mathrm{~h}$ & $4 \mathrm{~h}$ & $6 \mathrm{~h}$ & $8 \mathrm{~h}$ \\
\hline $1123 \mathrm{~K}$ & $27.3998 \pm 3.21$ & $42.1942 \pm 5.96$ & $53.009 \pm 5.48$ & $61.1964 \pm 10.9$ \\
\hline $1173 \mathrm{~K}$ & $43.5345 \pm 5.68$ & $67.0407 \pm 7.83$ & $84.223 \pm 9.07$ & $98.4523 \pm 15.6$ \\
\hline $1223 \mathrm{~K}$ & $68.0478 \pm 6.41$ & $104.790 \pm 11.6$ & $131.648 \pm 14.4$ & $153.889 \pm 18.3$ \\
\hline $1273 \mathrm{~K}$ & $105.574 \pm 12.9$ & $150.313 \pm 17.8$ & $184.504 \pm 20.8$ & $213.284 \pm 24.3$ \\
\hline
\end{tabular}

Tabla 2. Espesores de capa obtenidos experimentalmente. (Elaboración Propia)

En la Figura 21, se presentan los resultados de los espesores obtenidos de la medición de las capas boruradas en la fase $\mathrm{Fe}_{2} \mathrm{~B}$ para el proceso de borurización en polvo para la AISI 4150. De acuerdo con la Ecuación
(21) $\left(\mathrm{v}=2 \varepsilon D_{\mathrm{Fe}_{2} B}^{1 / 2} t^{1 / 2}=2 \varepsilon D_{\mathrm{Fe}_{2} B}^{1 / 2}\left(t_{\mathrm{v}}+t_{0}^{\mathrm{Fe}_{2} B}\right)^{1 / 2}\right)$, se graficó v v vs. $t$.

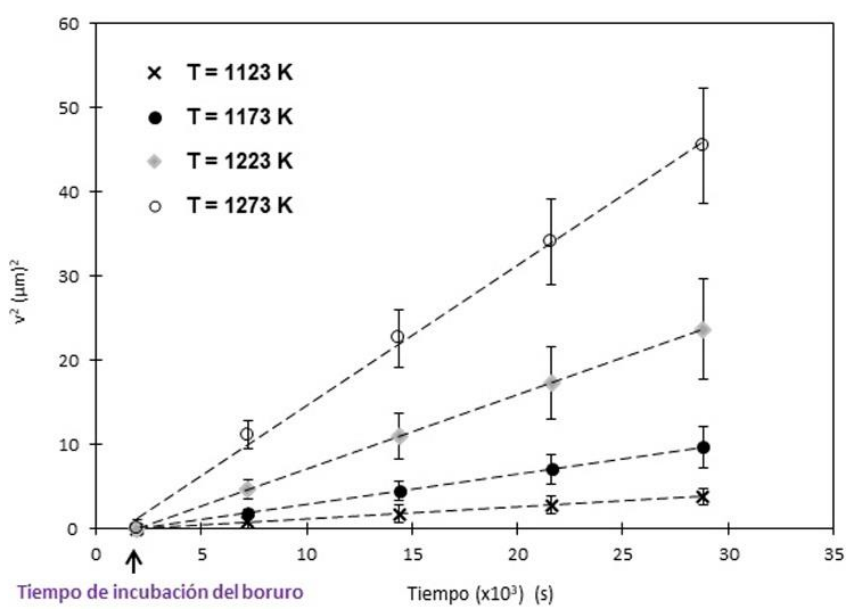

Figura 21. El cuadrado de los espesores $\left(\mathrm{v}^{2}\right)$ como función del tiempo de tratamiento $(t)$.

(Elaboración Propia)

En la Tabla (3) se muestran los valores numéricos de las pendientes los cuales se obtienen por cada línea recta de la Figura 21, así mismo, es posible calcular el valor de $\varepsilon^{2}\left(=C_{u p}^{\mathrm{Fe}_{2} B}-C_{l o w}^{\mathrm{Fe}_{2} B} / C_{l o w}^{\mathrm{Fe}_{2} B}-2 C_{0}+C_{u p}^{\mathrm{Fe}_{2} B}\right)$ a partir de los valores conocidos como $C_{u p}^{F e_{2} B}, C_{l o w}^{F e_{2} B}$ y $C_{0}$.

\begin{tabular}{|c|c|c|c|}
\hline Temperatura & Capa & $\varepsilon^{2}$ & $\begin{array}{c}4 \varepsilon D_{F_{2} B} \\
\left(\mathrm{~m}^{2} \mathrm{~s}^{-1}\right)\end{array}$ \\
\hline $1123 \mathrm{~K}$ & \multirow{4}{*}{$\mathrm{Fe}_{2} \mathrm{~B}$} & \multirow{4}{*}{$1.66945 \times 10^{-3}$} & $1.43 \times 10^{-13}$ \\
\hline $1173 \mathrm{~K}$ & & & $3.61 \times 10^{-13}$ \\
\hline $1223 \mathrm{~K}$ & & & $8.82 \times 10^{-12}$ \\
\hline $1273 \mathrm{~K}$ & & & $1.59 \times 10^{-12}$ \\
\hline
\end{tabular}

Tabla 3. Pendientes $\left(4 \varepsilon^{2} D_{\mathrm{Fe}_{2} \mathrm{~B}}\right.$ ), obtenidas de la Figura 21.

(Elaboración Propia)

Al momento de iniciar la difusión, los átomos de boro comienzan a incubar $\left(t_{0}^{\mathrm{Fe}_{2} \mathrm{~B}}\right)$ formando núcleos en toda la superficie del material, generando una película base, finalmente esta etapa termina cuando comienzan a aparecer los primeros boruros de hierro $\left(t_{\mathrm{v}}\right)$ como se muestra en la Figura (22). 


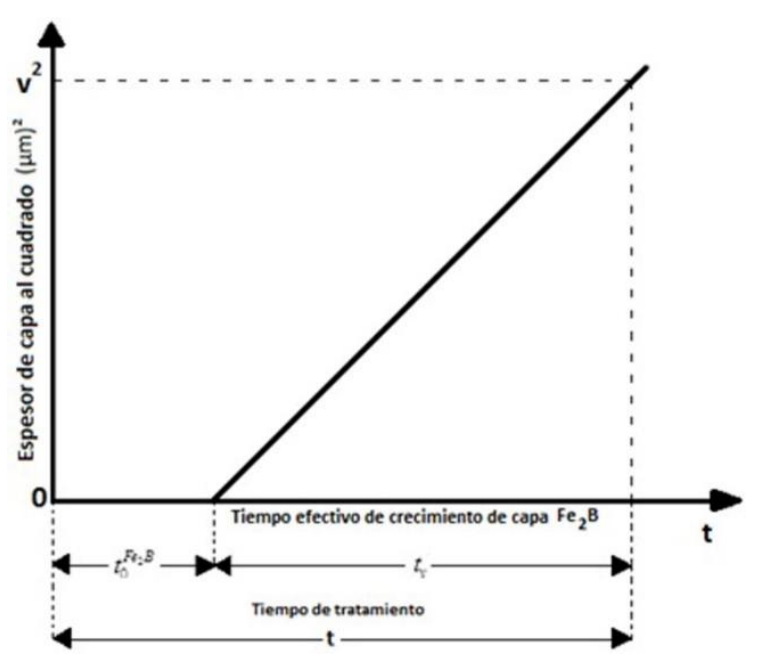

Figura 22. Representación del tiempo de tratamiento.

(Elaboración Propia)

El tiempo de incubación se puede observar en la intersección en el eje de las abscisas en la Figura 21. Este tiempo de incubación para el acero borurado AISI 4150 fue de $1950 \mathrm{~s}$, en el que es posible observar una pequeña capa delgada correspondiente a la fase $\mathrm{Fe}_{2} \mathrm{~B}$ como se muestra en la Figura 23.

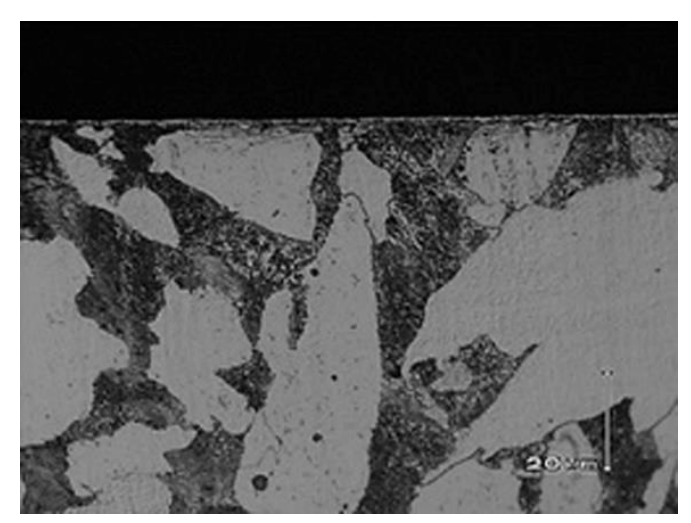

Figura 23. Sección transversal de acero AISI 4150 con $t_{0}^{F e_{2} B}=32.50 \mathrm{~min}$.

(Elaboración Propia)

Una vez obtenidos los espesores de capa y las pendientes es posible obtener los coeficientes de difusión respectivos a cada temperatura como se muestra en la Tabla (4).
(Elaboración Propia)

\begin{tabular}{|c|c|}
\hline $\begin{array}{c}\text { Temperatura } \\
\left(\mathrm{m}^{2} / \mathrm{s}\right)\end{array}$ & $\begin{array}{c}\text { Coeficiente de difusión }\left(D_{\mathrm{Fe}_{2} B}\right) \\
3.652 \times 10^{-12}\end{array}$ \\
\hline $1123 \mathrm{~K}$ & $9.221 \times 10^{-12}$ \\
\hline $1173 \mathrm{~K}$ & $2.253 \times 10^{-11}$ \\
\hline $1223 \mathrm{~K}$ & $4.061 \times 10^{-11}$ \\
\hline $1273 \mathrm{~K}$ & \\
\hline
\end{tabular}

Tabla 4. Coeficientes de difusión $\left(D_{\mathrm{Fe}_{2} \mathrm{~B}}\right)$.

Asumiendo un comportamiento tipo Arrhenius de los coeficientes de difusión tomados de la Tabla (4), la energía de activación $Q_{\mathrm{Fe}_{2} B}$ es la cantidad necesaria de energía para penetrar la superficie del acero AISI 4150. La energía de activación calculada fue de $193.458 \mathrm{kJmol}^{-}$ 1 y se obtiene a partir de la pendiente del gráfico representado en la Figura (24). El coeficiente de difusión vía relación de Arrhenius se puede expresar con la Ecuación 24.

$$
\begin{aligned}
& D_{F_{2} B}= \\
& 0.003768 \exp \left(-\frac{193.458 k J m o l^{-1}}{R T}\right) \quad\left(m^{2} s^{-1}\right) .
\end{aligned}
$$

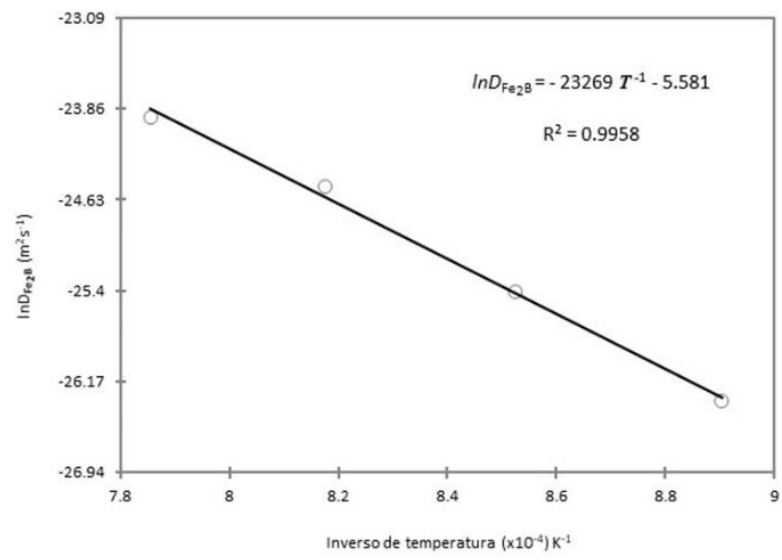

Figura 24. Las pendientes del gráfico de $\ln D_{\mathrm{Fe}_{2} B}$ vs $1 / T$ determinan la energía de activación $Q_{\mathrm{Fe}_{2} B}$.

(Elaboración Propia)

De la Ecuación (24), $R$ es la constante universal de los gases $(=8.314 \mathrm{~J} / \mathrm{molK})$, y $T$ representa la temperatura 
absoluta (K). El valor de la energía de activación ${ }^{*} Q_{\mathrm{Fe}_{2} B}$ indica la energía necesaria para estimular la difusión de boro a lo largo de la dirección cristalográfica [001] en la fase $\mathrm{Fe}_{2} \mathrm{~B}$. Continuando con el modelo de difusión, es posible estimar los espesores de capa con la técnica de borurización en polvo por medio de la Ecuación (25).

$$
\mathrm{v}_{\mathrm{Fe}_{2} B}=2 \varepsilon_{\mathrm{Fe}_{2} B} D_{0}^{1 / 2} e^{-\left(\frac{Q}{2 R T}\right)} t^{1 / 2}
$$

El modelo de difusión que se presenta en este trabajo, puede ser utilizado para estimar el espesor de capa borurada en diferentes aleaciones ferrosas. Los espesores aconsejables varían dependiendo de la aplicación. En el caso de dispositivos para el desprendimiento de viruta y herramentales se recomienda un espesor aproximado de 15 a $20 \mu \mathrm{m}$. Para aplicaciones sometidas a la extrusión de plásticos es aconsejable utilizar espesores de 50 a $250 \mu \mathrm{m}$.

\section{Validación experimental del modelo de difusión}

Para validar el modelo de difusión, se verificó con la comparación experimental de del espesor de la capa borurada obtenido a la temperatura de $1253 \mathrm{~K}$, durante 2.5 $\mathrm{h}$ de tratamiento con el espesor estimado por la Ec. (25). En la Figura (25), se muestra la microfotografía de la sección transversal de la muestra borurada a la temperatura de $1253 \mathrm{~K}$, con $2.5 \mathrm{~h}$ de tratamiento obtenida con la técnica de Microscopía Electrónica de Barrido, con una concentración de boro $C_{u p}^{F_{2} B}=60 \times 10^{3} \mathrm{~mol} / \mathrm{m}^{3}$ hacia el interior del substrato, finalizando con una concentración de boro $C_{\text {low }}^{\mathrm{Fe}_{2} B}=59.8 \times 10^{3} \mathrm{~mol} / \mathrm{m}^{3}$. El valor estimado teóricamente y el valor experimental son muy aproximados. Desde un punto de vista práctico, es decir, para optimizar y automatizar el proceso termoquímico de borurización, basta con sustituir el tiempo y temperatura de tratamiento en la Ecuación (25), para determinar el espesor de capa óptimo de la fase $\mathrm{Fe}_{2} \mathrm{~B}$.
*Un átomo que se difunde debe escurrirse entre los átomos circundantes para ocupar su nueva posición. El átomo que se

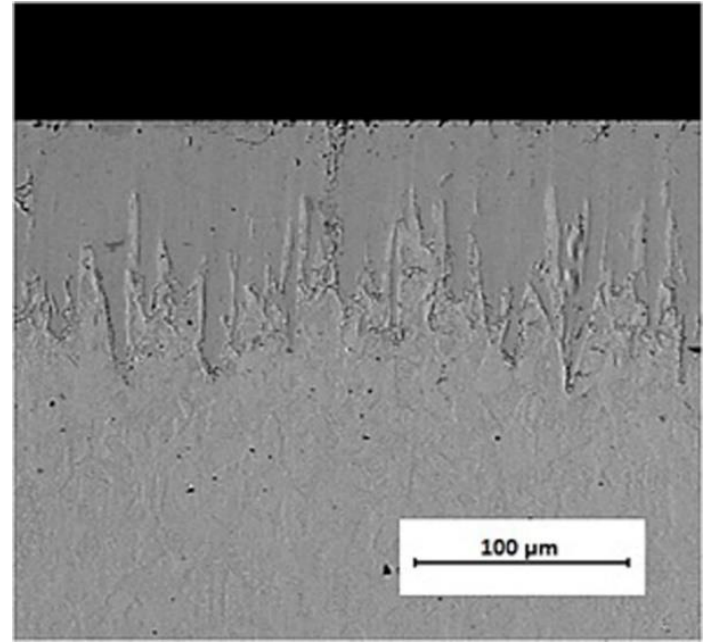

Figura 25. Microfotografía (SEM) de la capa $\mathrm{Fe}_{2} \mathrm{~B}$ formada sobra la superfice del acero AISI 4150 a la temperatura de $1253 \mathrm{~K}$, con un tiempo de tratamiento de $2.5 \mathrm{~h}$.

(Elaboración Propia)

\begin{tabular}{|c|c|c|c|c|}
\hline $\begin{array}{c}\text { Tem peratura } \\
(\mathrm{K})\end{array}$ & Capa & $\begin{array}{c}\text { Tiempo de } \\
\text { tratamiento } \\
(\mathrm{h})\end{array}$ & $\begin{array}{c}\text { Espesor de la capa } \\
\text { borurada estimada } \\
\text { con la Ecuación (25) }\end{array}$ & $\begin{array}{c}\text { Espesor } \\
\text { esperimental de la } \\
\text { capa borurada }\end{array}$ \\
\hline 1253 & $\mathrm{Fe}_{2} \mathbf{B}$ & 2.5 & 108.066 & $95.941 \pm 15.8965$ \\
\hline
\end{tabular}

Tabla 5. Comparación entre los valores de los espesores de capa de la fase $\mathrm{Fe}_{2} \mathrm{~B}$ obtenido a la temperatura de tratamiento de $1253 \mathrm{~K}$ con 2.5 h y el valor estimado por la Ecuación (25).

(Elaboración Propia)

\section{Conclusiones}

La cinética de crecimiento de las capas $\mathrm{Fe}_{2} \mathrm{~B}$ formadas en la superficie del acero AISI 4150 fue estimada para el proceso de borurización en polvo usando un modelo de difusión. El modelo considera la influencia del tiempo de incubación, tiempo y temperatura de tratamiento. La ley de crecimiento de las capas boruradas (ley de crecimiento parabólico) ha sido derivada puramente de consideraciones matemáticas a partir de la ecuación de balance de masa que a su vez se relaciona con las leyes fundamentales de Fick. Se determinó que la energía de activación para el crecimiento de una sola fase $\left(\mathrm{Fe}_{2} \mathrm{~B}\right)$ de alrededor de $193.458 \mathrm{kJmol}^{-1}$ con la técnica de borurización de polvo, la energía de activación $\left(Q_{F_{2 B}}\right)$ es

difunde, debe atravesar una barrera de energía potencial (energía de activación). 
una constante que identifica a cada substrato. Por un lado, se tiene que en el proceso de borurización base polvo, la reacción química a causa de las temperaturas de tratamiento, pueden ser de dos formas (ver Ecuaciones (22) y (23)):

$$
\begin{aligned}
\mathrm{B}_{4} \mathrm{C}+4 \mathrm{KBF}_{4}+3 \mathrm{SiC}+2 \mathrm{O}_{2} \rightarrow 8 \mathrm{~B}+4 \mathrm{KF}+3 \mathrm{SiF}_{4} \\
+4 \mathrm{CO} . \quad(22) \\
\mathrm{B}_{4} \mathrm{C}+4 \mathrm{KBF}_{4}+3 \mathrm{SiC}+3 \mathrm{O}_{2} \rightarrow 6 \mathrm{~B}+4 \mathrm{KF}+3 \mathrm{SiF}_{4}+4 \mathrm{CO} \\
+\mathrm{B}_{2} \mathrm{O}_{2} .
\end{aligned}
$$

La tasa de suministro de átomos de boro es de 8 a 6 , que también depende del control del oxígeno $\left(\mathrm{O}_{2}\right)$. Finalmente, el modelo de difusión desarrollado, permite que pueda ser utilizado para estimar los espesores de capas boruradas en diferentes aceros borurados para un conjunto de condiciones experimentales. Lo que permite facilitar la optimización y automatización del proceso de borurización de polvo (empaquetamiento en caja).

\section{Agradecimientos}

El trabajo descrito en el presente artículo fue financiado por las becas otorgadas por PRODEP y CONACyT.

\section{Referencias}

[1] Lajtín Y., Arzamásov B. (1987). Tratamiento químico-térmico de los metales. Moscú, Rusia, Mir.

[2] Uslu., H. Comert., M. Ipek., O. Ozdemir., y C. Bindal. (2005) Evaluation of borides forme don AISI P20 Steel. Materials and Design, 28 (2007), 55-6.

[3] M. González Fernández de Castro, Boración de aceros. Tesis de Doctorado, Universidad Politécnica de Madrid, España, 1999.

[4] O. A. Bravo Bárcenas, Endurecimiento superficial de una aleación base Fe-Cr-Ni: cinética de crecimiento y caracterización mecánica de capas boruradas. Tesis de Doctorado, Instituto Politécnico Nacional, México, 2011.

[5] E. Meléndez, I. Campos, E. Rocha, M. A. Barron, Structural and strength characterization of steels subjected to boriding thermochemical process, Materials Science and Engineering, A234-236 (1997) 900-903.

[6] Tapia Quintero, Estimación de la cinética de crecimiento de los boruros $\mathrm{FeB}$ y Fe2B en aceros para herramienta de corte AISI M2. Tesis de Maestría, Instituto Politécnico Nacional, México, 2010.

[7] Ehrenberg. (2013), Acero SISA P20 AISI P20 Tool Steel, México DF: Aceros SISA. Recuperado de: http://sisa1.com.mx/pdf/Acero\%20SISA\%20P20.pdf

[8] Valued Customer. (2005), P20.doc, Ohio, US: Cincinnati Tool Steel Company. Recuperado http://cintool.com/catalog/mold_quality/P20.pdf

[9] F. Guzmán Ornelas, Análisis de Aceros por Microscopia Óptica. Tesis de Ingeniería, Instituto Politécnico Nacional, México, 2013.

[10] C. M. Brakman, A. W. J. Gommers, and E. J. Mittemeijer, Bonding of $\mathrm{Fe}$ and $\mathrm{Fe}-\mathrm{C}, \mathrm{Fe}-\mathrm{Cr}$, and $\mathrm{Fe}-\mathrm{Ni}$ alloys; Boride-layer growth kinetics, J. Mater. Res., 4 (1989) 1354-1370.
[11] G. Vandér Voort, (1999), Metalography Principles and Practice, Ohio, EU, ASTM International Materials.

[12] C. Kittel. (1997). Introducción a la física del estado sólido. Barcelona, España, Reverté.

[13] O. A. Bravo Bárcenas, Endurecimiento superficial de una aleación base Fe-Cr-Ni: cinética de crecimiento y caracterización mecánica de capas boruradas. Tesis de Doctorado, Instituto Politécnico Nacional, México, 2011.

[14] M. Ortiz Domínguez, Contribución de la modelación matemática en el tratamiento termoquímico de borurización. Tesis de Doctorado, SEPIESIME Zacatenco, Instituto Politécnico Nacional, México, 2013.

[15] G. Ramírez Sandoval, Caracterización de grietas auto-afines en capas borurizadas para aceros estructurales AISI 1045. Tesis de Maestría, SEPI-ESIME Zacatenco, Instituto Politécnico Nacional, México, 2006.

[16] C. I. Villa Velázquez Mendoza, Estudio del agrietamiento tipo palmqvist y evaluación de esfuerzos residuales en aceros borurados AISI 1018. Tesis de Doctorado, Instituto Politécnico Nacional, México, 2009. 